\title{
miR-23a-3p inhibits sepsis-induced kidney epithelial cell injury by suppressing Wnt/ $\beta$-catenin signaling by targeting wnt5a
}

\author{
Junwei $Y e^{1 * \bullet}$, Huibing Feng ${ }^{2 \star \bullet}$, and Zhiyong Peng ${ }^{1 \oplus \star}$ \\ ${ }^{1}$ Department of Critical Care Medicine, Zhongnan Hospital of Wuhan University, Wuhan, Hubei, China \\ ${ }^{2}$ Department of Critical Care Medicine, Huangshi Central Hospital of Edong Healthcare Group, Hubei Polytechnic University, \\ Huangshi, Hubei, China
}

\begin{abstract}
The present study was designed to investigate the involvement of miR-23a-3p in the progression of sepsis-induced acute kidney injury (AKI). The expression levels of miR-23a-3p and wnt5a in sepsis-induced AKI patients and lipopolysaccharide (LPS)-treated HK-2 cells were detected by real-time PCR and western blotting. Then, the effects of miR-23a-3p overexpression on cell viability, apoptosis, and inflammatory cytokines secretion in LPS-stimulated HK-2 cells were investigated. Moreover, luciferase reporter assay was performed to confirm the regulatory relationship between miR-23a-3p and wnt5a. Whether miR$23 a-3 p$ regulated the activation of $\mathrm{Wnt} / \beta$-catenin signaling was also explored. $\mathrm{mR}-23 a-3 p$ was lowly expressed in the serum of patients with sepsis-associated AKI and in LPS-treated HK-2 cells. In addition, the overexpression of miR-23a-3p restrained LPS-induced proliferation inhibition and promotion of apoptosis and cytokine production in HK-2 cells. Moreover, wnt5a was identified as a target of miR-23a-3p, which could be negatively regulated by miR-23a-3p. Overexpression of miR-23a-3p suppressed the activation of $\mathrm{Wnt} / \beta$-catenin signaling in LPS-treated HK-2 cells, which was markedly reversed by wnt5a upregulation. Upregulation of miR-23a-3p may alleviate LPS-induced cell injury by targeting wnt5a and inactivating Wnt/ $\beta$ catenin pathway, which may serve as a novel therapeutic target for sepsis-associated AKI.
\end{abstract}

Key words: Acute kidney injury; miR-23a-3p; sepsis; Wnt/ $\beta$-catenin pathway; wnt5a

\section{Introduction}

Sepsis is a systemic inflammatory condition caused by the invasion of pathogens, leading to fatal multiple organ dysfunction syndrome (MODS) $(1,2)$. The kidney is one of the most vulnerable target organs when sepsis occurs. Approximately $50 \%$ of patients with sepsis will develop acute kidney injury (AKI) that is characterized by the rapid decline and even loss of kidney function (3). Accordingly, $\mathrm{AKI}$ is a life-threatening complication of sepsis, which is implicated in increased morbidity and mortality in the intensive care unit $(4,5)$. It is widely accepted that there are different pathophysiological mechanisms that lead to AKI (4). Many urinary and serum biomarkers have been described as increased in kidney injury, such as cystatin $C$, kidney injury molecule-1, among others, but they are not effective in reflecting kidney damage (6). In recent years, research has proposed that the release of inflammatory mediators, kidney hemodynamic abnormality, as well as microcirculation dysfunction and kidney cell apoptosis caused by kidney injury are involved in the pathogenesis of sepsis-associated $\operatorname{AKI}(7,8)$. Therefore, it is critical to identify potential biomarkers for early diagnosis, treatment, and prognosis of septic AKI.

Micro (mi) RNAs are a class of small non-coding RNAs composed of 19-25 nucleotides, which serve as posttranscriptional regulators of gene expression by binding to the target mRNA and inhibiting its translation (9). Since miRNA was first discovered in nematodes by Lee et al. (10), tens of thousands of miRNAs have been identified, and over $60 \%$ of human protein-coding genes are regulated by miRNAs (11). In mammals, miRNAs are widely involved in a variety of biological processes, such as cell differentiation, substance metabolism, tumor growth, and immune response, which are expected to become novel targets for the treatment of diverse diseases (12). In recent years, an extensive body of evidence suggests the involvements of several miRNAs in the progress of sepsis

Correspondence: Zhiyong Peng <zhiyongpeng@webmailcn.com>

${ }^{*}$ These authors contributed equally to this study. 
$(13,14)$. For instance, Ge et al. (15) reported that miR-23a$3 p$ expression was significantly downregulated in both the sepsis-induced AKI and sepsis-non AKI patients compared with the healthy controls. Nevertheless, to our knowledge, the physiopathological role of miR-23a-3p in sepsis-associated AKI remains elusive. It would be interesting to understand the relationship of miRNAs and mitochondrial oxidative stress and endothelial dysfunctions in sepsis and if they are related to early stages of this condition. The present study aimed to analyze the influence of miR-23a-3p in sepsis patients with AKI, and further investigate its regulatory pathway in lipopolysaccharide (LPS)-associated injury in kidney tubular epithelial cells.

\section{Material and Methods}

\section{Patients and controls}

Twenty-five sepsis patients with AKI (mean age $=45.75$ years, 15 females and 10 males) who fulfilled the definition and diagnostic criteria for sepsis proposed by the Society of Critical Care Medicine (SCCM) and the European Society of Intensive Care Medicine (ESICM) were enrolled in this study. The criteria for AKI were based on the Acute Kidney Injury Network (AKIN) classification $(16,17)$. Patients were included if they: 1) were over 18 years old; 2) conformed to the diagnostic criteria of SCCM/ ESICM; and 3) conformed to the diagnostic criteria of AKIN. The exclusion criteria were as follows: 1) patients aged less than 18 years or more than 80 years; 2) pregnant females; 3) patients with malignant tumors; 4) patients with chronic liver and kidney diseases, autoimmune diseases, and blood system diseases; and 5) patients treated with systemic hormones and immunosuppressive agents. In addition, 20 healthy volunteers (mean age $=44.39$ years, 12 females and 8 males) were recruited as healthy controls. The characteristics of the participants enrolled in the study are shown in Table 1. Written informed consents were obtained prior to blood sample collection. The experiments were performed with the approval of the Ethics Review Committee of Zhongnan Hospital of Wuhan University.

\section{Cell culture, treatment, and transfection}

Human kidney tubular epithelial cell line HK-2 purchased from Cell Bank of Chinese Academy of Sciences (China) was cultured in Dulbecco's modified Eagle's medium (DMEM) supplemented with $10 \%$ fetal bovine serum, L-glutamine, $100 \mathrm{U} / \mathrm{mL}$ penicillin, and $100 \mathrm{mg} / \mathrm{mL}$ streptomycin (all from Gibco, USA) in humidified air with $5 \% \mathrm{CO}_{2}$ at $37^{\circ} \mathrm{C}$. At $70 \%$ confluence, HK-2 cells were treated with $1 \mu \mathrm{g} / \mathrm{mL}$ lipopolysaccharide (LPS; Sigma, USA) for $24 \mathrm{~h}$ to induce the in vitro model of sepsis, as previously described $(18,19)$. The miR-23a-3p mimics (miR-23a-3p), mimic scrambled control (miR-NC), antimiR-23a-3p oligonucleotides, anti-miR negative control (anti-miR-NC), pcDNA3.1-wnt5a (wnt5a), and pcDNA3.1 empty vector (Vector) were bought from GenePharma (China). Cell transfection was performed using Lipofectamine 2000 reagents (Invitrogen, USA) before LPS stimulation according to manufacturer's instructions. Transfection efficiency was assessed by real-time PCR and western blotting.

\section{Luciferase reporter assay}

The wild-type or mutated wnt5a expression luciferase plasmids were co-transfected with miR-23a-3p mimics or miR-NC (negative control) into HK-2 cells using Lipofectamine 2000. After $48 \mathrm{~h}$ transfection, the dual-luciferase reporter system (Promega, USA) was used to detect the luciferase activity.

\section{Reverse transcription and quantitative polymerase chain reaction (RT-qPCR)}

Trizol reagent (Invitrogen) was used to extract total RNA according to the manufacturer's instructions. PrimeScript RT reagent kit (Takara, China) was then applied for first-strand cDNA synthesis. The mRNA expression levels were detected by qPCR using the SYBR Green kit (Takara). The primers were miR-23a-3p (Forward: $5^{\prime}$-CCA ATTGCGCCTTCAGGCTA-3', Reverse: 5'-CGGCAGAGT CCTTACCCACA-3'); wnt5a (Forward: 5'-ATTCTTGGT GGTCGCTAGGTA-3', Reverse: 5'-CGCCTTCTCCGATG TACTGC-3'), GAPDH (Forward: 5'-CGCGAGAAGATGA CCCAGAT-3', Reverse: 5'-GGGCATACCCCTCGTAGAT

Table 1. Baseline characteristics of sepsis-induced acute kidney injury (AKI) patients $(n=25)$ and healthy individuals $(n=20)$.

\begin{tabular}{lccc}
\hline Baseline characteristics & Sepsis-induced AKI & Healthy controls & P value \\
\hline Age (years) & $45.75 \pm 3.21$ & $44.39 \pm 2.54$ & $>0.05$ \\
Sex (males/females) & $15 / 10$ & $12 / 8$ & \\
Body mass $\left(\mathrm{kg} / \mathrm{m}^{2}\right)$ & $26.23 \pm 7.54$ & $25.99 \pm 9.28$ & $>0.05$ \\
Blood urea nitrogen $(\mathrm{mM})$ & $10.25 \pm 1.56$ & $5.23 \pm 1.77$ & $<0.05$ \\
Serum creatinine $(\mathrm{mM})$ & $260.23 \pm 20.56$ & $46.75 \pm 17.53$ & $<0.05$ \\
\hline
\end{tabular}

Data are reported as means $\pm S D$, except for sex $(n)$. Groups were compared with the Student's $t$-test. 
G-3'); and U6 (Forward: 5'-TCACTCTCAGAAGATCA-3', Reverse: 5'-GGGACGGACACGGTTG-3'). The results were normalized to GAPDH or U6, and the expression fold-change was calculated using the $2^{-\Delta \Delta C t}$ method.

\section{Cell viability assay}

Cell viability was determined using the CCK-8 kit (Dojindo Molecular Technologies, China). In brief, HK-2 cells were seeded onto 96-well plates at a density of $5 \times 10^{3}$ cells per well and incubated with $10 \mu \mathrm{L}$ CCK- 8 solution at 0 and $24 \mathrm{~h}$. The absorbance was then measured at $450 \mathrm{~nm}$ after $1 \mathrm{~h}$ incubation using a microplate reader (Bio-Rad Laboratories, USA).

\section{Cell apoptosis assay}

Cell apoptosis was measured by flow cytometry with Annexin V-FITC/PI double staining. HK-2 cells were collected and washed with cold PBS buffer. Flow cytometry analysis was performed using Annexin V-FITC/PI apoptosis detection kit (eBioscience, USA). The positive cells were considered as apoptotic cells.

\section{Immunoblotting}

The protein concentration was determined using a BCA protein assay kit (Thermo Scientific, China). Equal amounts of total protein were separated by SDS-PAGE, electroblotted on the polyvinylidene fluoride membranes (Millipore, USA), and immunoblotted with the primary antibodies (1:1000 dilution) against wnt5a, Bax, Bcl-2, cleaved caspase-3, $\beta$-catenin, c-myc, cyclin D1, and $\beta$-actin as a loading control, which were all obtained from Cell Signaling Technology (USA). The membranes were then incubated with horseradish peroxidase-conjugated IgG antibodies for $1 \mathrm{~h}$ at room temperature. The protein levels were measured using an enhanced chemiluminescence kit (Millipore) through ImageJ software (NIH, USA).

\section{ELISA}

The concentrations of interleukin (IL)- $6, \mathrm{IL}-1 \beta$, and tumor necrosis factor (TNF)- $\alpha$ in the culture supernatants were determined using commercial enzyme-linked immunosorbent assay (ELISA) kits (R\&D Systems, USA) following the manufacturer's protocol. The absorbance was measured at $450 \mathrm{~nm}$ on a microplate reader. The caspase-3 activity was determined with the Caspase-3 Colorimetric Activity Assay kit (Keygen Biotech, China). Absorbance values at $405 \mathrm{~nm}$ were recorded.

\section{Statistical analysis}

Data are reported as means $\pm S D$ and analyzed by SPSS software v19.0 (IBM, USA). All data were checked for normality using Kolmogorov-Smirnov tests. Comparisons were performed using Student's t-test and one-way ANOVA. Correlation between expression levels of
miR-23a-3p and wnt5a were performed using Spearman's rank correlation coefficient. Differences with $\mathrm{P}<0.05$ (twotailed) were considered statistically significant.

\section{Results}

\section{miR-23a-3p was downregulated in sepsis patients and LPS-treated HK-2 cells}

The expression levels of miR-23a-3p were initially measured in sepsis patients with $\mathrm{AKI}$ and healthy subjects using real-time PCR. The results demonstrated that miR$23 a-3 p$ expression was significantly decreased in serum of patients with sepsis-induced AKI compared to healthy controls (Figure 1A). miR-23a-3p expression was also suppressed in LPS-treated HK-2 cells compared to the untreated cells (Figure 1B).

\section{Overexpression of miR-23a-3p alleviated LPS-induced apoptosis and inflammation in HK-2 cells}

To explore the function of miR-23a-3p in LPS-treated HK-2 cells, we established a miR-23a-3p overexpression model in HK-2 cells by transfection with miR-23a-3p mimics prior to LPS stimulation, and the upregulation efficacy was confirmed by real-time PCR (Figure 2A). The effects of miR-23a-3p overexpression on cell proliferation, apoptosis, and inflammatory response in LPS-stimulated HK-2 cells were further determined. The results of CCK- 8 cell viability assay showed that compared to the untreated HK-2 cells, LPS treatment reduced the cell viability, while the overexpression of miR-23a-3p markedly increased the viability of LPS-stimulated HK-2 cells (Figure 2B). The treatment of HK-2 cells with LPS produced higher apoptotic rate as evidenced by the results of flow cytometry, while upregulation of miR-23a-3p significantly attenuated cell apoptosis (Figure 2C). Western blot analysis indicated that LPS-treated HK-2 cells showed higher levels of proapoptotic proteins Bax and cleaved caspase-3 and lower levels of antiapoptotic protein $\mathrm{Bcl}-2$ than those of untreated cells, while the enforced
A

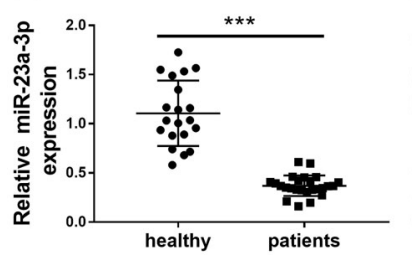

B

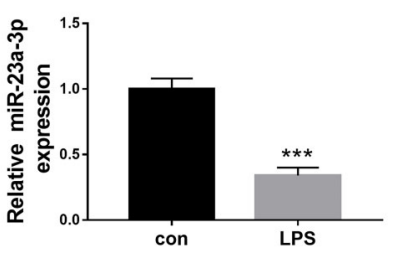

Figure 1. miR-23a-3p is downregulated in sepsis patients and lipopolysaccharide (LPS)-treated HK-2 cells. A, The expression of miR-23a-3p in serum of patients with sepsis-induced acute kidney injury $(n=25)$ and healthy controls $(n=20)$ was detected by RT-qPCR. $B$, The expression of miR-23a-3p in LPS-treated $(1 \mu \mathrm{g} / \mathrm{mL} ; 24 \mathrm{~h})$ HK-2 cells and untreated cells (con) was detected by RT-qPCR. Data are reported as means $\pm S D$. ${ }^{* \star} P(0.001$ (Student's $t$-test). 
A

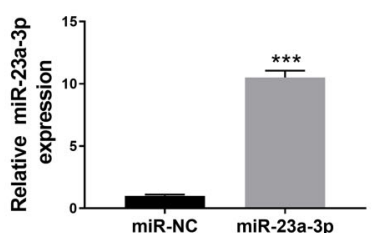

D
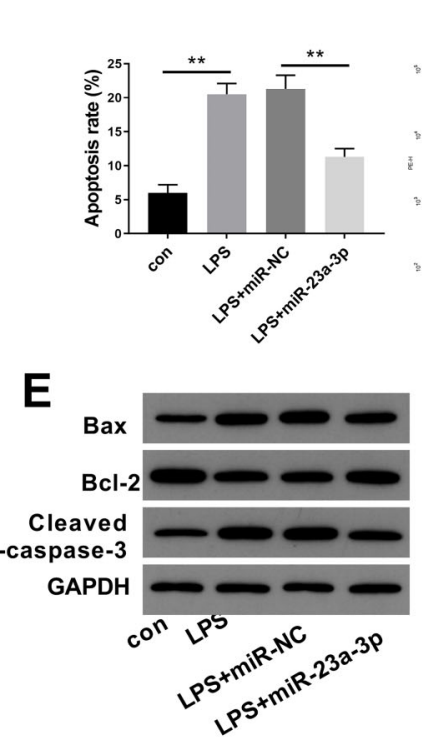

B

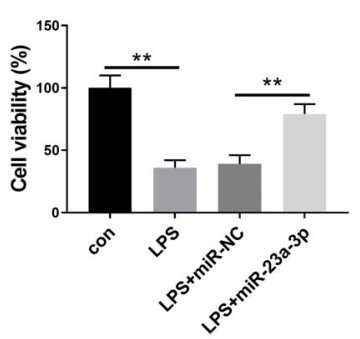

con
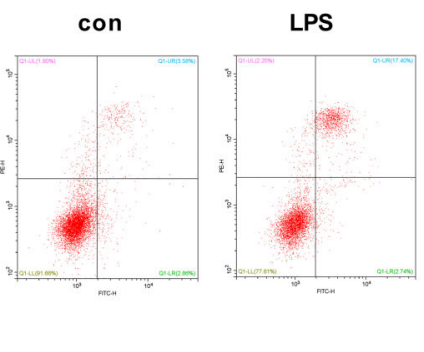

C

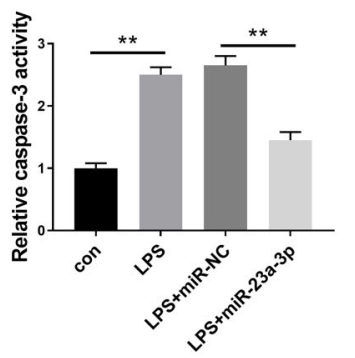

LPS+miR-NC LPS+miR-23a-3p

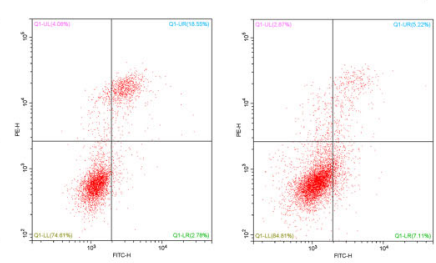

$\mathbf{F}$

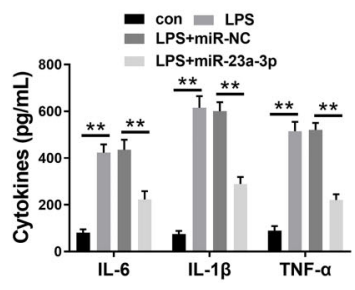

Figure 2. Overexpression of miR-23a-3p alleviates lipopolysaccharide (LPS)-induced apoptosis and inflammation in HK-2 cells. A, RTqPCR was conducted to detect the expression levels of miR-23a-3p in HK-2 cells after the overexpression of miR-23a-3p. B, CCK-8 assay was used to detect the proliferation of HK-2 cells induced by LPS after the overexpression of miR-23a-3p. C, The colorimetric assay was carried out to assess the caspase-3 activity in HK-2 cells induced by LPS after the overexpression of miR-23a-3p. D, Flow cytometry was applied to evaluate the apoptotic rate of HK-2 cells induced by LPS after the overexpression of miR-23a-3p. E, Western blotting was done to detect the protein levels of Bax, Bcl-2, and cleaved caspase-3 in HK-2 cells induced by LPS after the overexpression of miR-23a-3p. F, ELISA was performed to estimate the production of interleukin (IL)-6, IL-1 $\beta$, and tumor necrosis factor (TNF)- $\alpha$ in HK-2 cells induced by LPS after the overexpression of miR-23a-3p. The results are representative of three independent experiments ( $n=3$ per group). Data are reported as means $\pm \mathrm{SD}$. ${ }^{*} \mathrm{P}<0.05$; ${ }^{* *} \mathrm{P}<0.01$; ${ }^{* * *} \mathrm{P}<0.001$ (ANOVA). con: control; NC: negative control.

expression of miR-23a-3p exhibited reverse effects of the protein levels of these apoptosis-associated proteins (Figure 2D). Consistently, the colorimetric assay revealed that enhanced caspase- 3 activity was observed in LPStreated HK-2 cells, which was inhibited by miR-23a-3p overexpression (Figure 2E).

ELISA indicated that LPS treatment induced higher inflammatory cytokines, including IL-6, IL-1 $\beta$, and TNF- $\alpha$ in HK-2 cells, which was significantly reversed by miR23a-3p upregulation (Figure 2F).

\section{miR-23a-3p directly targeted and negatively regulated} wnt5a in HK-2 cells

We next elucidated the possible downstream targets of miR-23a-3p. Based on starBase software (https//bio.tools), the predicted binding sequences of miR-23a-3p and wnt5a is shown in Figure $3 \mathrm{~A}$. Luciferase assay in HK-2 cells showed that the luciferase activities of wnt5a were significantly reduced by miR-23a-3p mimics (Figure $3 B$ ). As expected, the mRNA and protein levels of wnt5a were significantly downregulated in miR-23a-3p-overexpressed HK-2 cells (Figure $3 \mathrm{C}$ and D). HK-2 cells were transfected with $\mathrm{miR}-23 a-3 p$ inhibitor to suppress the miR-23a-3p expression. Subsequent experiments revealed that miR$23 a-3 p$ knockdown led to the upregulation of wnt5a expression at mRNA and protein levels (Figure $3 E$ and $F)$. The mRNA expression of wnt5a was significantly upregulated (Figure $3 G$ ) and negatively correlated with miR23a-3p expression (Figure $3 \mathrm{H}$ ) in serum of sepsis-induced AKI patients. 
A

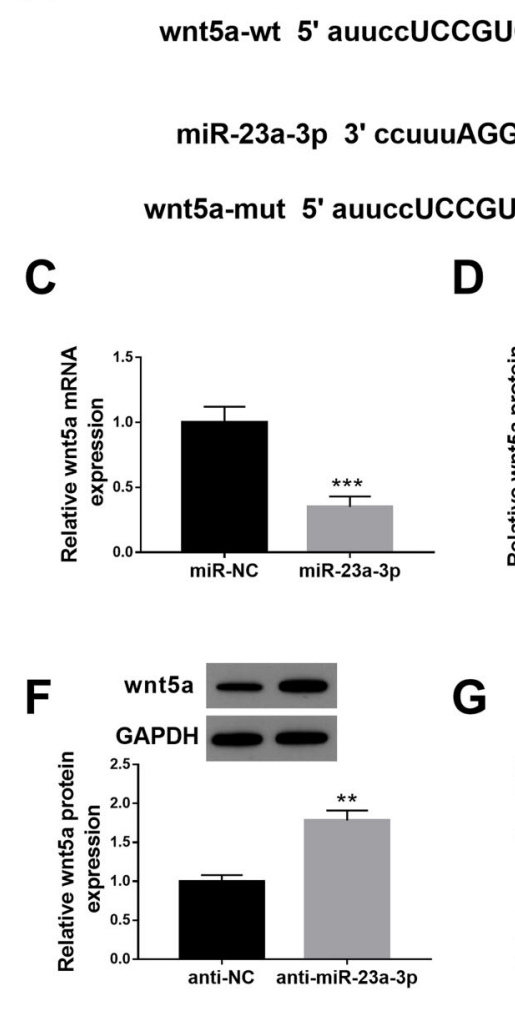

B

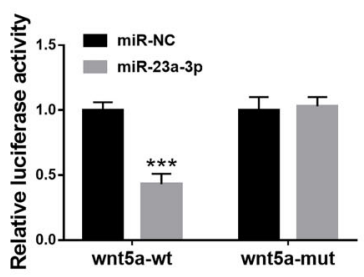

E
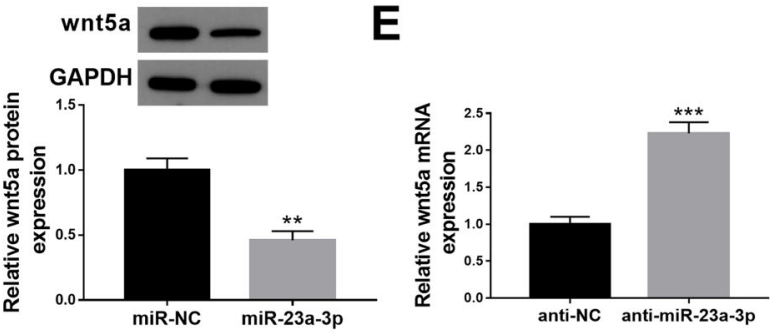

H

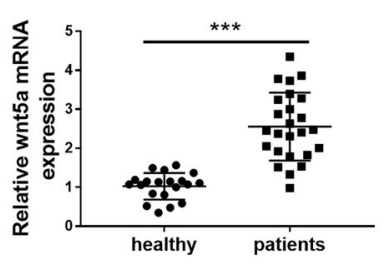

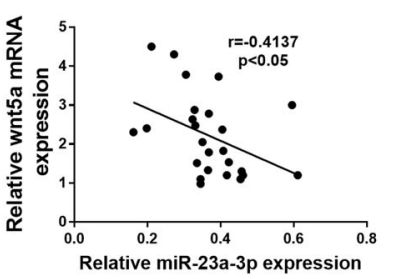

Figure 3. miR-23a-3p directly targets and negatively regulates wnt5a in HK-2 cells. A, The base complementary pairing relationship between miR-23a-3p and wnt5a. B, Dual luciferase assay was performed to determine the targeting relationship of miR-23a-3p and wnt5a. C and D, RT-qPCR and western blotting were performed to detect the mRNA and protein levels of wnt5a in HK-2 cells after the overexpression of miR-23a-3p. E and F, RT-qPCR and western blotting were performed to detect the expression of wnt5a in HK-2 cells after the knockdown of miR-23a-3p. G. The mRNA expression of wnt5a in serum of patients with sepsis-induced AKI $(n=25)$ and healthy controls $(n=20)$ was detected by RT-qPCR. H, The correlation between miR-23a-3p and wnt5a expression in serum of patients with sepsis-induced acute kidney injury was analyzed using Spearman's rank correlation coefficient. The results are representative of three independent experiments ( $\mathrm{n}=3$ per group). Data are reported as means $\pm S D$. ${ }^{* *} \mathrm{P}<0.01$; ${ }^{* * *} \mathrm{P}<0.001$ (Student's $t$-test). NC: negative control; wt: wild-type; mut: mutated.

\section{miR-23a-3p attenuated LPS-induced HK-2 cell injury by downregulating wnt5a}

To further investigate whether the influence of miR-23a-3p on LPS-induced cell injury was achieved by regulating the expression of wnt5a in HK-2 cells, the expression of wnt5a was successfully overexpressed by transfection with pcDNAwnt5a as demonstrated by western blotting (Figure 4A). The co-overexpression of miR-23a-3p and wnt5a significantly reversed the effects of miR-23a-3p overexpression on cell viability (Figure 4B), apoptotic rate (Figure 4C), expression of apoptosis-related proteins (Figure 4D), caspase-3 activity (Figure 4E), and the production of cytokines (Figure 4F) in LPS-stimulated HK-2 cells. These results suggested that miR23a-3p upregulation enhanced LPS-induced injury in HK-2 cells by the suppression of wnt5a.
miR-23a-3p inhibited the activation of Wnt/ $\mathrm{\beta}-$ catenin signaling pathway in LPS-treated HK-2 cells

On the basis of the critical role of the $\mathrm{Wnt} / \beta$-catenin signaling pathway in kidney injury, we examined whether miR-23a-3p inactivated $W n t / \beta$-catenin signaling pathway. The protein levels of $\mathrm{Wnt} / \beta$-catenin signaling pathwayrelated proteins including $\beta$-catenin, c-myc, and cyclin D1 were then detected. Western blotting analysis consistently confirmed that the expression levels of $\beta$-catenin, c-myc, and cyclin D1 were elevated in LPS-treated HK-2 cells, whereas the restoration of miR-23a-3p expression inhibited the activation of $\mathrm{Wnt} / \beta$-catenin signaling, which was significantly reversed after wnt5a overexpression (Figure 5A and B). 
A

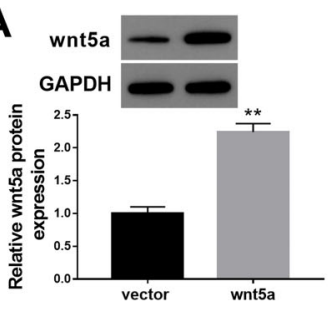

D

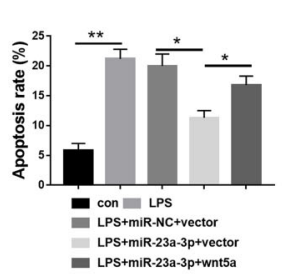

E

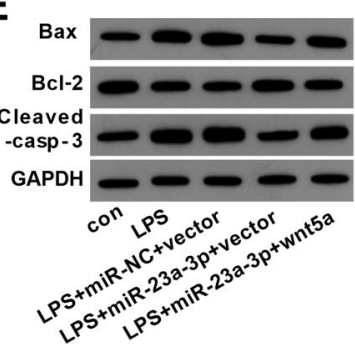

B

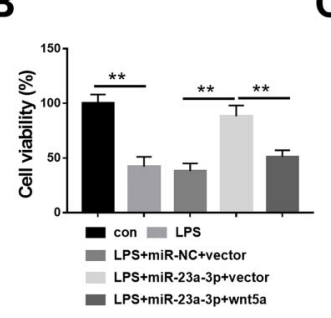

C

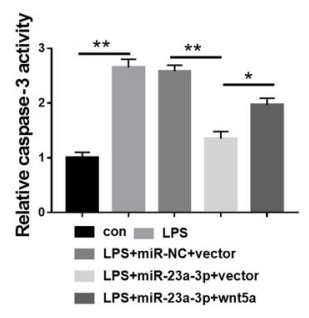

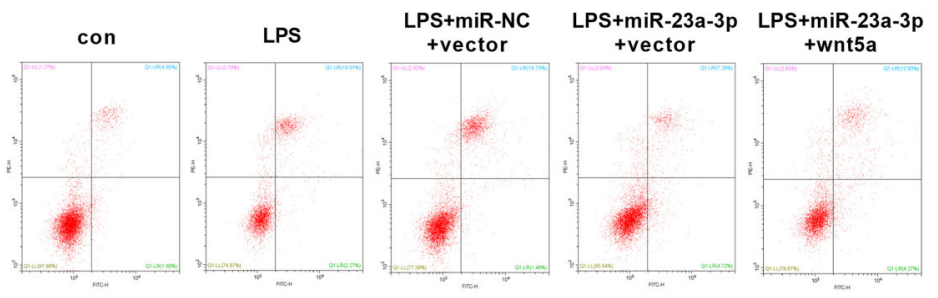
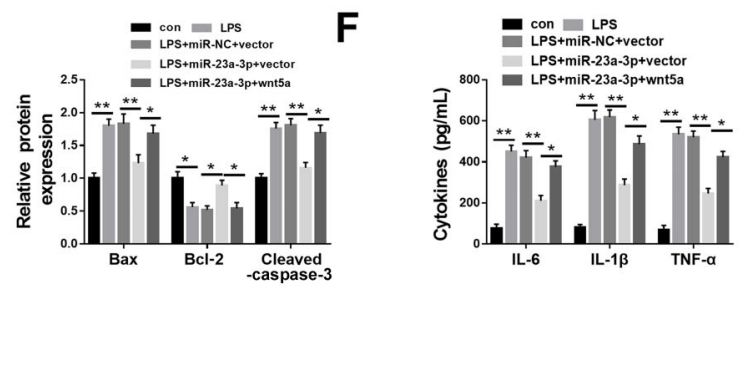

Figure 4. miR-23a-3p attenuates lipopolysaccharide (LPS)-induced HK-2 cell injury by downregulating wnt5a. A, Western blotting was conducted to detect the protein levels of wnt5a in HK-2 cells after the overexpression of wnt5a. Cell viability (B), caspase-3 activity (C), apoptosis rate (D), the expression of apoptosis-related proteins (E), and the production of cytokines (F) were determined in LPSstimulated HK-2 cells after the overexpression of miR-23a-3p and wnt5a. The results are representative of three independent experiments ( $n=3$ per group). Data are reported as means $\pm S D$. ${ }^{*} \mathrm{P}<0.05$; ${ }^{* *} \mathrm{P}<0.01$ (ANOVA). con: control; NC: negative control.

\section{Discussion}

The results of the current study showed that miR-23a$3 p$ expression was suppressed in patients diagnosed with sepsis-associated AKI and in LPS-induced HK-2 cells. In addition, overexpression of miR-23a-3p alleviated LPS-associated injury in HK-2 cells. Our results further revealed the interaction between miR-23a-3p and wnt5a, and miR-23a-3p overexpression restrained LPS-induced cell injury by suppression of wnt5a expression. Finally, we demonstrated that miR-23a-3p upregulation could mediate LPS-associated cell injury by inactivating $\mathrm{Wnt} / \beta$ catenin signaling pathway.

Recently, accumulating evidence has strongly implied that a few miRNAs have been characterized as diagnostic biomarkers in the pathogenesis of sepsis and related complications, including miR-21 (20), miR-214 (21), and miR-122 (22). Microarray assay has shown that miR-23a$3 p$ was significantly downregulated in sepsis-induced AKI
A

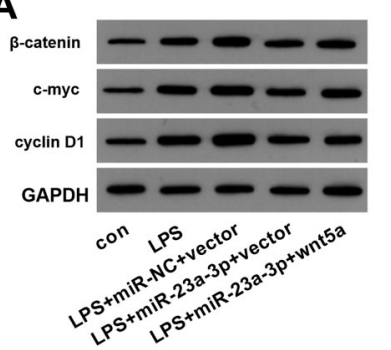

B

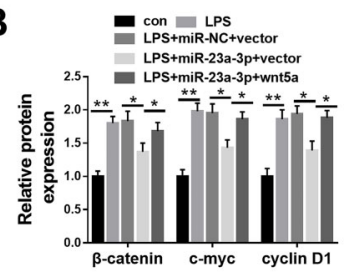

Figure 5. miR-23a-3p inhibits the activation of Wnt/ $\beta$-catenin signaling pathway in lipopolysaccharide (LPS)-treated HK-2 cells. $\mathbf{A}$ and $\mathbf{B}$, Western blotting was conducted to detect the protein levels of $\beta$-catenin, c-myc, and cyclin D1 in LPS-stimulated HK-2 cells after the overexpression of miR-23a-3p and wnt5a. The results are representative of three independent experiments $(n=3$ per group). Data are reported as means $\pm S D$. ${ }^{*} \mathrm{P}<0.05$; ${ }^{* *} \mathrm{P}<0.01$ (ANOVA). con: control; NC: negative control. 
patients compared with the healthy controls. In previous reports, miR-23a-3p has been reported to be abnormally expressed in diverse tumor types and possesses oncogenic or tumor-suppressing functions. For example, Ma et al. (23) demonstrated that miR-23a-3p mitigated mucosal melanoma growth, migration, invasion, and tumorigenicity by targeting ADCY1 via the suppression of CAMP and MAPK signaling pathways. Chen et al. (24) showed that miR-23a-3p inhibited the proliferation and invasion of oral squamous cell carcinomas cells, while inducing apoptosis by decreasing FGF2 expression. Xiang et al. (25) reported that miR-23a-3p might target $\mathrm{PCDH} 17$ to accelerate the proliferation and G1/S cell cycle transition in hepatocellular carcinoma. The present study demonstrated for the first time that the expression of miR-23a-3p was significantly downregulated in serum of patients with sepsis-induced AKI and LPS-induced septic AKI cell model. These data suggested that the aberrant expression of miR-23a-3p might be associated with the progression of septic AKI.

The pathogenesis of septic AKI is associated with the apoptosis of kidney tubular epithelial cells and inflammatory injury (26-28). Consistently, this research implied that LPS treatment inhibited cell viability and increased apoptosis and the levels of inflammatory cytokines IL-6, IL-1 $\beta$, and TNF- $\alpha$ in HK-2 cells, while miR-23a-3p

\section{References}

1. Napolitano LM. Sepsis 2018: definitions and guideline changes. Surg Infect (Larchmt) 2018; 19: 117-125, doi: 10. 1089/sur.2017.278.

2. Salomão R, Ferreira BL, Salomão MC, Santos SS, Azevedo LCP, Brunialti MKC. Sepsis: evolving concepts and challenges. Braz J Med Biol Res 2019; 52: e8595, doi: 10.1590/ 1414-431x20198595.

3. Poston JT, Koyner JL. Sepsis associated acute kidney injury. BMJ 2019; 364: k4891, doi: 10.1136/bmj.k4891.

4. Zarbock A, Gomez H, Kellum JA. Sepsis-induced acute kidney injury revisited: pathophysiology, prevention and future therapies. Curr Opin Crit Care 2014; 20: 588-595, doi: $10.1097 / \mathrm{MCC} .0000000000000153$.

5. Gómez H, Kellum JA. Sepsis-induced acute kidney injury. Curr Opin Crit Care 2016; 22: 546-553, doi: 10.1097/MCC. 0000000000000356.

6. Medic B, Rovcanin B, Vujovic KS, Obradovic D, Duric D, Prostran M. Evaluation of novel biomarkers of acute kidney injury: the possibilities and limitations. Curr Med Chem 2016; 23: 1981-1997, doi: 10.2174/0929867323666160210130256.

7. Emlet DR, Shaw AD, Kellum JA. Sepsis-associated AKI: epithelial cell dysfunction. Semin Nephrol 2015; 35: 85-95, doi: 10.1016/j.semnephrol.2015.01.009.

8. Zhao W, Zhang L, Chen R, Lu H, Sui M, Zhu Y, et al. SIRT3 protects against acute kidney injury via AMPK/mTORregulated autophagy. Front Physiol 2018; 9: 1526, doi: 10.3389/fphys.2018.01526.

9. Lu TX, Rothenberg ME. MicroRNA. J Allergy Clin Immunol 2018; 141: 1202-1207, doi: 10.1016/j.jaci.2017.08.034. upregulation repressed these biological behaviors of HK-2 cells under LPS-stimulated conditions. These results showed that miR-23a-3p might be implicated in the occurrence and development of septic AKI by regulating cell apoptosis and inflammation.

Further investigations were performed to elucidate the downstream mechanism of miR-23a-3p in sepsis-induced AKI. Bioinformatics and dual luciferase analysis confirmed that wnt5a, a noncanonical Wnt ligand, was a direct target of miR-23a-3p, the expression of which was negatively correlated with miR-23a-3p expression in patients with septic AKI. It has been demonstrated that Wnt5a plays an emerging role in hyperuricemia-induced kidney tubular injury (29). Also, Wnt5a possesses pro-inflammatory properties in the pathogenesis of inflammatory diseases (30), including sepsis $(31,32)$. Inspired by the miRNA regulatory network, we observed that the overexpression of wnt5a could reverse the effects of miR-23a-3p in HK-2 cells treated with LPS. Further studies demonstrated that targeting of wnt5a by miR-23a-3p resulted in the inhibition of Wnt/ $\beta$-catenin axis in LPS-treated HK-2 cells.

We concluded that miR-23a-3p suppressed the development of sepsis-induced AKI by downregulating $\mathrm{Wnt} / \beta$ catenin pathway, at least in part, via targeting wnt5a. Our findings might provide a novel therapeutic strategy for sepsis-associated complications.

10. Lee RC, Feinbaum RL, Ambros V. The C. elegans heterochronic gene lin-4 encodes small RNAs with antisense complementarity to lin-14. Cell 1993; 75: 843-854, doi: 10.1016/0092-8674(93)90529-Y.

11. Xu K, Lin J, Zandi R, Roth JA, Ji L. MicroRNA-mediated target mRNA cleavage and $3^{\prime}$-uridylation in human cells. Sci Rep 2016; 6: 30242, doi: 10.1038/srep30242.

12. Vishnoi A, Rani S. MiRNA biogenesis and regulation of diseases: an overview. Methods Mol Biol 2017; 1509: 1-10, doi: 10.1007/978-1-4939-6524-3.

13. Kingsley SMK, Bhat BV. Role of microRNAs in sepsis. Inflamm Res 2017; 66: 553-569, doi: 10.1007/s00011-0171031-9.

14. Benz F, Roy S, Trautwein C, Roderburg C, Luedde T. Circulating microRNAs as biomarkers for sepsis. Int $\mathrm{J} \mathrm{Mol}$ Sci 2016; 17: 78, doi: 10.3390/ijms17010078.

15. Ge QM, Huang CM, Zhu XY, Bian F, Pan SM. Differentially expressed miRNAs in sepsis-induced acute kidney injury target oxidative stress and mitochondrial dysfunction pathways. PloS One 2017; 12: e0173292, doi: 10.1371/journal. pone.0173292.

16. Koza Y. Acute kidney injury: current concepts and new insights. J Inj Violence Res 2016; 8: 58-62, doi: 10.5249/jivr. v8i1.610.

17. Kang JS, Moon KH, Youn YH, Park JS, Ko SH, Jeon YS. Factors associated with postoperative acute kidney injury after hip fractures in elderly patients. J Orthop Surg (Hong Kong) 2020; 28: 2309499019896237, doi: 10.1177/230949 9019896237. 
18. Shen J, Liu L, Zhang F, Gu J, Pan G. LncRNA TapSAKI promotes inflammation injury in HK-2 cells and urine derived sepsis-induced kidney injury. J Pharm Pharmacol 2019; 71: 839-848, doi: 10.1111/jphp.13049.

19. Wang J, Song J, Li Y, Shao J, Xie Z, Sun K. Down-regulation of LncRNA CRNDE aggravates kidney injury via increasing MiR-181a-5p in sepsis. Int Immunopharmacol 2020; 79: 105933, doi: 10.1016/j.intimp.2019.105933.

20. Fu D, Dong J, Li P, Tang C, Cheng W, Xu Z, et al. MiRNA-21 has effects to protect kidney injury induced by sepsis. Biomed Pharmacother 2017; 94: 1138-1144, doi: 10.1016/ j.biopha.2017.07.098.

21. Ge C, Liu J, Dong S. miRNA-214 protects sepsis-induced myocardial injury. Shock 2018; 50: 112-118, doi: 10.1097/ SHK.0000000000000978.

22. Abou El-Khier NT, Zaki ME, Alkasaby NM. Study of microRNA-122 as a diagnostic biomarker of sepsis. Egypt J Immunol 2019; 26: 105-116.

23. Ma M, Dai J, Tang H, Xu T, Yu S, Si L, et al. MicroRNA-23a$3 p$ inhibits mucosal melanoma growth and progression through targeting adenylate cyclase 1 and attenuating cAMP and MAPK pathways. Theranostics 2019; 9: 945-960, doi: $10.7150 /$ thno.30516.

24. Chen F, Qi S, Zhang X, Wu J, Yang X, Wang R. miR-23a-3p suppresses cell proliferation in oral squamous cell carcinomas by targeting FGF2 and correlates with a better prognosis: miR-23a-3p inhibits OSCC growth by targeting FGF2. Pathol Res Pract 2019; 215: 660-667, doi: 10.1016/j. prp.2018.12.021.

25. Xiang $Y$, Yang $Y$, Lin C, Wu J, Zhang X. MiR-23a-3p promoted G1/S cell cycle transition by targeting protocadherin 17 in hepatocellular carcinoma. J Physiol Biochem 2020; 76: 123-134, doi: 10.1007/s13105-020-00726-4.

26. Havasi A, Borkan SC. Apoptosis and acute kidney injury. Kidney Int 2011; 80: 29-40, doi: 10.1038/ki.2011.120.

27. Zhang S, Li R, Dong W, Yang H, Zhang L, Chen Y, et al. RIPK3 mediates renal tubular epithelial cell apoptosis in endotoxininduced acute kidney injury. Mol Med Rep 2019; 20: 1613-1620, doi: 10.3892/mmr.2019.10416.

28. Zheng $\mathrm{C}$, Zhou $\mathrm{Y}$, Huang $\mathrm{Y}$, Chen $\mathrm{B}, \mathrm{Wu} \mathrm{M}$, Xie $\mathrm{Y}$, et al. Effect of ATM on inflammatory response and autophagy in renal tubular epithelial cells in LPS-induced septic AKI. Exp Ther Med 2019; 18: 4707-4717, doi: 10.3892/etm.2019. 8115.

29. Setyaningsih WAW, Arfian N, Suryadi E, Romi MM, Tranggono U, Sari DCR. Hyperuricemia induces Wnt5a/ Ror2 gene expression, epithelial-mesenchymal transition, and kidney tubular injury in mice. Iran J Med Sci 2018; 43: 164-173, doi: 10.3892/etm.2019.8115.

30. Pashirzad M, Shafiee M, Rahmani F, Behnam-Rassouli R, Hoseinkhani F, Ryzhikov M, et al. Role of Wnt5a in the pathogenesis of inflammatory diseases. J Cell Physiol 2017; 232: 1611-1616, doi: 10.1002/jcp.25687.

31. Villar J, Cabrera-Benitez NE, Ramos-Nuez A, Flores C, Garcia-Hernandez S, Valladares F, et al. Early activation of pro-fibrotic WNT5A in sepsis-induced acute lung injury. Crit Care 2014; 18: 568, doi: 10.1186/s13054-014-0568-z.

32. Schulte DM, Kragelund D, Muller N, Hagen I, Elke G, Titz A, et al. The wingless-related integration site-5a/secreted frizzled-related protein- 5 system is dysregulated in human sepsis. Clin Exp Immunol 2015; 180: 90-97, doi: 10.1111/ cei.12484. 